\title{
Performance of the autonomous PLATO Antarctic Observatory over two full years
}

\author{
Daniel M. Luong-Van ${ }^{* a}$, Michael C. B. Ashley ${ }^{\mathrm{a}}$, Xiangqun Cui ${ }^{\mathrm{b}}$, Jon R. Everett ${ }^{\mathrm{a}}$, Longlong Feng ${ }^{\mathrm{c}}$, \\ Xuefei Gong ${ }^{\mathrm{b}}$, Shane Hengst ${ }^{\mathrm{a}}$, Jon S. Lawrence, ${ }^{\mathrm{d}, \mathrm{e}}$, John W. V. Storey ${ }^{\mathrm{a}}$, Lifan Wang ${ }^{\mathrm{c}, \mathrm{f}}$, \\ Huigen Yang ${ }^{\mathrm{g}}$, Ji Yang ${ }^{\mathrm{c}}, \mathrm{Xu} \mathrm{Zhou}^{\mathrm{h}}$, Zhengxi Zhu ${ }^{\mathrm{c}}$ \\ ${ }^{a}$ School of Physics, University of New South Wales, Sydney NSW 2052, Australia. \\ ${ }^{\mathrm{b}}$ Nanjing Insititute of Astronomical Optics \& Technology, Nanjing 210042, China. \\ ${ }^{c}$ Purple Mountain Observatory, Nanjing 210008, China. \\ ${ }^{\mathrm{d}}$ Department of Physics and Engineering, Macquarie University, NSW 2109, Australia. \\ eAnglo-Australian Observatory, NSW 1710, Australia. \\ fPhysics Department, Texas A\&M University, College Station, TX 77843. \\ ${ }^{\mathrm{g}}$ Polar Research Institute of China, Pudong, Shanghai 200136, China. \\ ${ }^{\mathrm{h}}$ National Astronomical Observatories, Chinese Academy of Science, Beijing 100012, China.
}

\begin{abstract}
For continuous observation at locations that are inhospitable for humans, the desirability of autonomous observatories is self evident. PLATO, the 'PLATeau Observatory' was designed to host an easily configurable instrument suite in the extremely cold conditions on the Antarctic plateau, and can provide up to $1 \mathrm{~kW}$ of power for the instruments. Powered by jet fuel and the Sun, PLATO and its instruments have been taking nearly uninterrupted astronomical science and sitetesting data at Dome A, the coldest, highest and driest location ${ }^{1}$ on the Antarctic Plateau, since their deployment by the 24th Chinese expedition team in January 2008. At the time of writing, PLATO has delivered a total uptime of 730 days. Following a servicing mission by the 25 th Chinese expedition team in 2008-9, PLATO has achieved $100 \%$ up-time (520 days) and has been in continuous contact with the rest of the world via its Iridium satellite modems. This paper discusses the performance of the observatory itself, assesses the sources of energy and dissects how the energy is divided between the core observatory functions of instrument power, heating, control and communication.
\end{abstract}

Keywords: Antarctica, remote power, robotics, site-testing

\section{INTRODUCTION}

The Antarctic Plateau offers the astronomy community one of the best locations for ground based observations ${ }^{1,2,3}$. Over the past two and a half years, the PLATO observatory has been collecting site testing and scientific data at Dome A, the highest location on the Antarctic Plateau. Data from PLATO and the CHINARE/AAD Automated Weather Station have shown that Dome A is not only one of the driest locations on Earth, but also has the lowest wind speed and lowest ground-layer turbulence height. These unique conditions present attractive opportunities for the development of some of the most capable radio, terahertz, infrared and optical ground-based astronomical installations.

With an elevation of $\sim 4100 \mathrm{~m}$ and $1300 \mathrm{~km}$ from the coast, Dome A is one of the most remote locations on Earth. Nevertheless, it can be reached by overland traverse, allowing for the transport of considerable amounts of cargo. The Polar Research Institute of China (PRIC) 2008 traverse, carried out as part of the "PANDA" IPY program, provided the logistical and technical support to both transport and install PLATO. In subsequent years, the Chinese summer traverse team has continued to service and upgrade the PLATO observatory and instrumentation. Presently there is no capability for year-round occupation at Dome $\mathrm{A}$ and the need for continuous scientific data ${ }^{4,5,6,7}$, including in the middle of winter, justifies the requirement of a fully robotic, autonomous observatory.

*daniel@phys.unsw.edu.au 
PLATO is the third generation of autonomous Antarctic observatories built by the University of New South Wales. Powered by solar power during periods of sufficient light, and Jet-A1 or similar fuels when there is not, PLATO provides a maximum of $1000 \mathrm{~W}$ of electrical power for scientific instrumentation, heating, control and continuous satellite communication. This paper provides an analysis of the PLATO observatory as a whole, evaluating its performance in its core responsibilities of power generation and delivery, thermal support and communication. A more thorough description of the physical layout, electronics and control strategy, has previously been published ${ }^{8,9}$.

\section{POWER SOURCES}

PLATO derives the majority of its power from two banks of three single-cylinder diesel engines. The six engines are identical $350 \mathrm{~cm}^{3}$ displacement Hatz 1B30s that directly drive three-phase brushless generators, the output of which is then diode rectified to DC. In the first year, as a risk-mitigation measure, two of the engines were instead fitted with high-efficiency brushed DC motors used as generators. These performed comparably to the alternators, but after the first servicing mission the engines were all fitted with alternators to simplify maintenance. Each engine can provide a maximum of $1200 \mathrm{~W}$ and multiple engines can be run together for more power. The standard mode of operation is to run one engine at a time. Automated oil and auxiliary management systems extend the maintenance interval of the engines to allow for the engine bank to provide a year or more of operation between servicing missions.

During summer, two arrays of three solar-panels provide a maximum of $1000 \mathrm{~W}$ when sunlight is available. One array points North East and the other North West to increase the duration of maximum solar power. In the summer of 2008 the panels were connected to two maximum-power-point-trackers (MPPT) that optimize the loading on the solar panels to extract the maximum available energy. These units proved to be unsuitable for remote operation, so during the first servicing mission they were replaced by a simple, shunt-regulated controller. The shunt regulator is, however, only rated for two panels, so four of the panels are presently unused. Unfortunately, a heavy snow drift during the winter of 2009 placed so much weight on the solar panel cables that they became disconnected and solar power was not available until the summer 2009-10 servicing mission.

Figure 1 shows the total power generation. The results have processed by a three-day moving-window low-pass filter (unless noted) to allow for a more general overview of the system performance over the multi-year time scale. Note the reduced solar output after the first year and the lack of solar power during the sunrise of 2009. The period of no power after mid-winter 2008 was when PLATO ceased operation due to a large exhaust leak in the engine module.

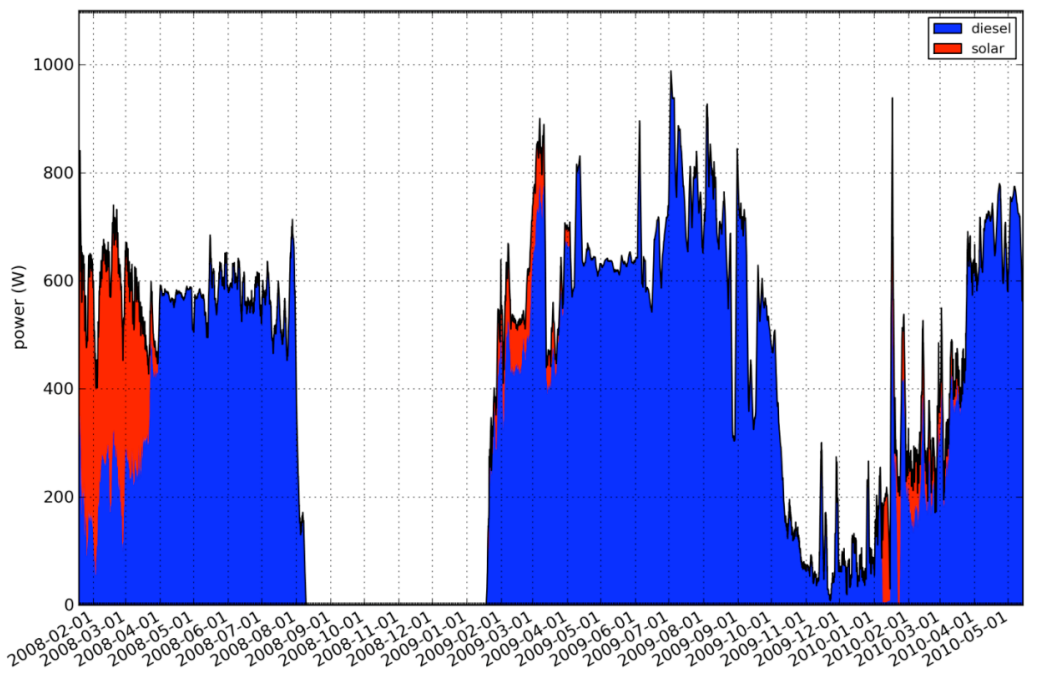

Figure 1. Total PLATO power consumption and breakdown between diesel and solar power. (color available online) 


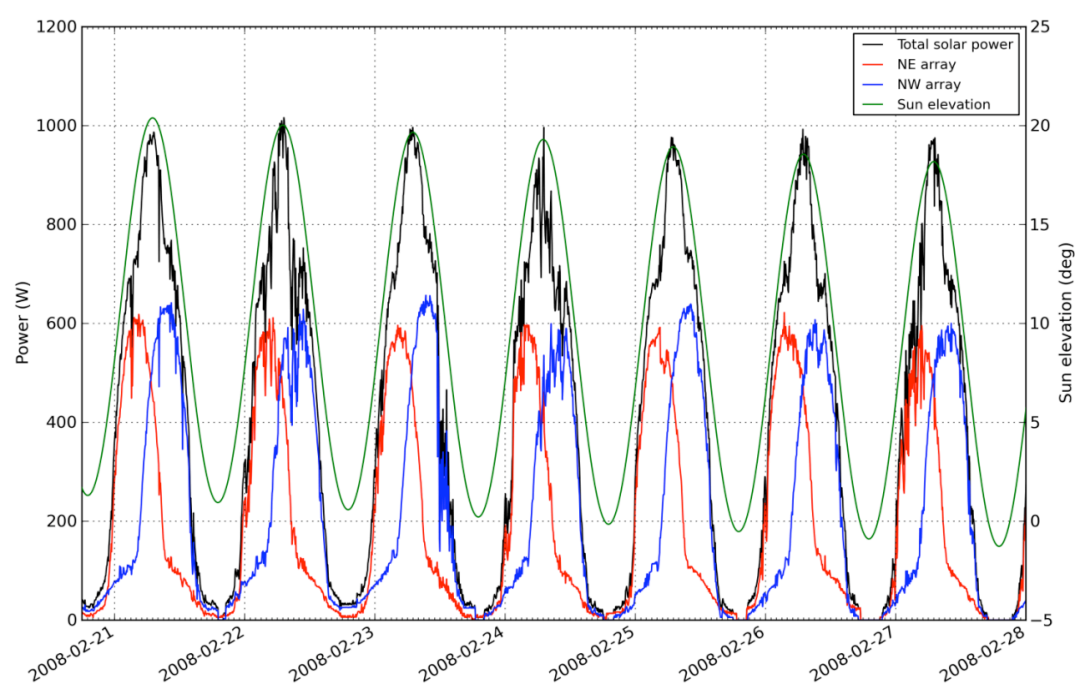

Figure 2. Solar power generated over a one-week period in 2008 during the setting of the Sun. The red trace is the North East array, the blue is the North West array and the black is the total solar power. The green line shows the Sun elevation. (color available online)

Figure 2 shows the profile of solar power generation in 2008. The peak occurred just before sunset as this was when there was when there was significant instrument and heating power demand and the sun was face-on to the vertically mounted panels. In 2008 PLATO used two arrays, each of three panels, with each of the two arrays individually connected to its own MPPT. Before the Sun began to set, there was on average 12.3 hours per day when more than $500 \mathrm{~W}$ of solar power was available. The average power before the Sun began to set was $387 \mathrm{~W}$. The highly reflective yet irregular surface of the snow very effectively diffuses the sunlight, reducing the need for tracking the panels directly at the Sun. This is evident from the more than $20 \mathrm{~W}$ of power available when the Sun was at an elevation of only $0.5^{\circ}$, and directly behind the panels. When the MPPT was no longer available from 2009 onwards, the two panels and lack of an MPPT reduced the average power to $100 \mathrm{~W}$ during periods of high sunlight. The use of a shunt regulator reduced the solar panel output by approximately $30 \%$ compared to what would have been achieved with an MPPT.

\section{POWER CONSUMPTION}

The power consumption in PLATO can be divided into five broad categories that effectively cover where the energy and the valuable byproduct of heat is distributed. 'Internal instruments' are instruments and support computers internal to the instrument module. Any power in this category is also directly contributing to heating. 'External instruments' are instruments outside either of the modules and these instruments do not provide any useful heating to the instrument module. The scientific and site-testing instruments are the primary reason for PLATO and are considered to be the most useful consumers of energy. 'Internal heating' means the supplementary heaters inside the module that are used to keep the internal temperature above $-20^{\circ} \mathrm{C}$. External instruments frequently require supplementary 'External heating' to remain at their designed operating temperature; this energy also does not contribute to the warming of the instrument module. 'Engine housekeeping' is power used in the engine module to control the engines and related hardware; the heat from this is not required as there is already a slight excess of heat present due to the operation of the engines themselves. The useful heat from the high-voltage DC power conversion is discussed separately in Section 4.

The waste heat from the engines is not discussed in detail as it is not directly recoverable for instrument consumption. However, it performs the essential function of heating the main fuel tanks and keeping the exhaust hot enough to reduce the condensation of volatiles. The need to keep the engine module warm is the reason for the continued running of the engines during summer, albeit at a lower power output.

Figure 3 shows the power consumption of the PLATO observatory and where it was consumed over the past two and a half years. Graphically it can clearly be seen that more power is being directed into instrumentation in the later years; 
this is due to the evolving scientific suite and improved control techniques. One other contributing factor in the increase in available instrument power is the improved thermal performance of the module after 2009. Before 2009, two major instruments were mounted through the insulated roof of the instrument module, increasing its thermal conductivity. These two instruments were removed during the 2008 - 9 servicing mission and replaced with insulating plugs.

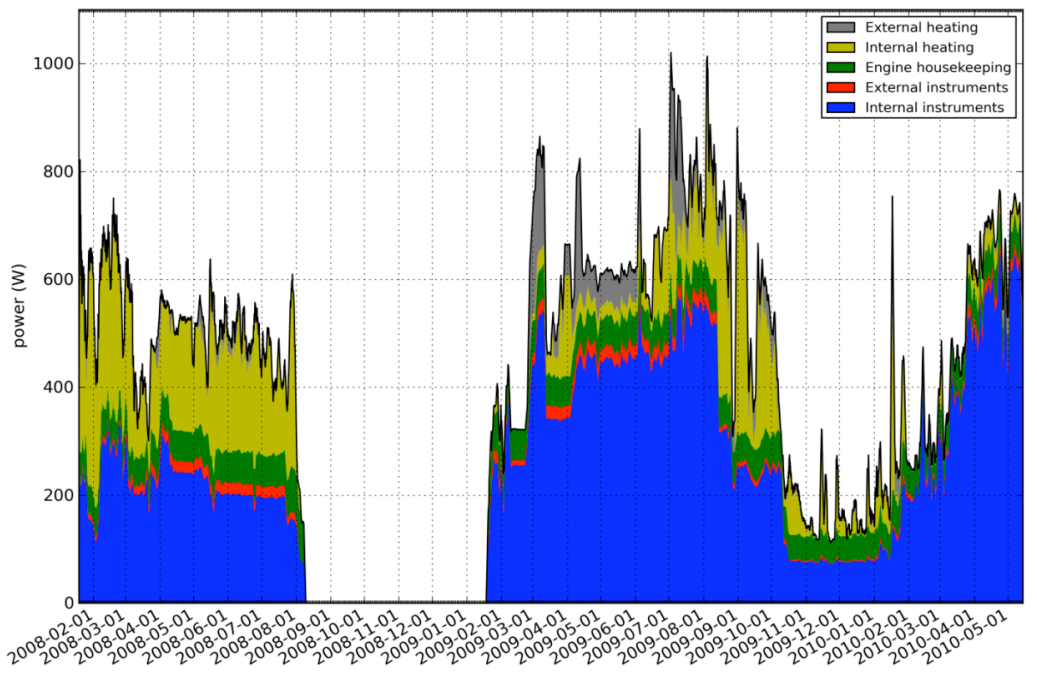

Figure 3. Itemized PLATO power consumption. (color available online)

Table 1 shows the integrated power consumption over each year of the Dome A campaign. Note the desirable very high internal instrument consumption during 2010. This higher fractional consumption in 2010 is due to an improved control strategy and the fact that PLATO is now supporting higher power instruments, which also contribute to internal heating. This value should be roughly constant as the winter progresses as the primary science instruments (which require total darkness) have only been recently turned on at the time of writing. They will be turned off when the Sun rises. For a review of the instrument complement in PLATO refer to Yang et al (2009) ${ }^{10}$.

Table 1. Itemized power consumption comparing different campaign years and instrument payloads

\begin{tabular}{|l|l|l|l|l|}
\hline & \multicolumn{1}{|c|}{$\mathbf{2 0 0 8 - 2 0 0 9}$} & \multicolumn{1}{|c|}{$\mathbf{2 0 0 9 - 2 0 1 0}$} & $\mathbf{2 0 1 0 - J u n e ~ 2 0 1 0}$ & \multicolumn{1}{|c|}{ Total } \\
\hline Internal instruments & $43.6 \%$ & $61.9 \%$ & $76.3 \%$ & $59.4 \%$ \\
\hline External instruments & $3.3 \%$ & $3.0 \%$ & $2.7 \%$ & $3.1 \%$ \\
\hline Engine housekeeping & $11.1 \%$ & $10.8 \%$ & $11.9 \%$ & $11.1 \%$ \\
\hline Internal heating & $38.9 \%$ & $15.0 \%$ & $5.9 \%$ & $20.1 \%$ \\
\hline External heating & $3.0 \%$ & $9.3 \%$ & $3.2 \%$ & $6.3 \%$ \\
\hline
\end{tabular}

\section{PLATO THERMAL PERFORMANCE}

The thermal performance of the instrument module is of great importance as all its heat must be derived from electricity during periods of no direct solar heating. The reason for this is that it must be placed at some distance from the engine module so that the exhaust does not interfere with the any instrumentation. The thermal performance of the PLATO engine module does not need to be as high as that of the instrument module, because of the large amount of waste heat available. For a discussion of the design considerations of a two-module design, see Lawrence et al (2009) ${ }^{8}$. 
Figure 4 shows the thermal losses from the instrument module through the winter of 2009. This period was chosen as it is the crucial time for instrument operation and the thermal balance is not misleadingly affected by solar radiation. The average thermal conductivity for this period was $11.9 \mathrm{~W} / \mathrm{K}$, which closely matches the design value of $12 \mathrm{~W} / \mathrm{K}$. The variation in thermal conductivity may be explained by the weather; the most strongly contributing factor is the wind speed which modifies the forced-convection heat transfer. As part of the recent instrument package a high-accuracy anemometer was fitted, but there is not yet a full winter period of data for analysis. For the instrument module in the middle of winter, of the order of $400-500 \mathrm{~W}$ is required to keep the module between -10 to $-20^{\circ} \mathrm{C}$.

All the electrical power that is fed from the engine module to the instrument module passes through DC/DC converters that convert from $120 \mathrm{~V}$ down to the $28 \mathrm{~V}$ main power bus. The efficiency of these converters is quite modest, typically around $80 \%$. However, they are mounted inside the instrument module and so any heat produced directly contributes to heating the internal instruments and lead-acid batteries. As can be seen in Figure 3 and Table 1, a small amount of internal heating is still required at all times; this essentially means that the efficiency of the power converters is of no importance.

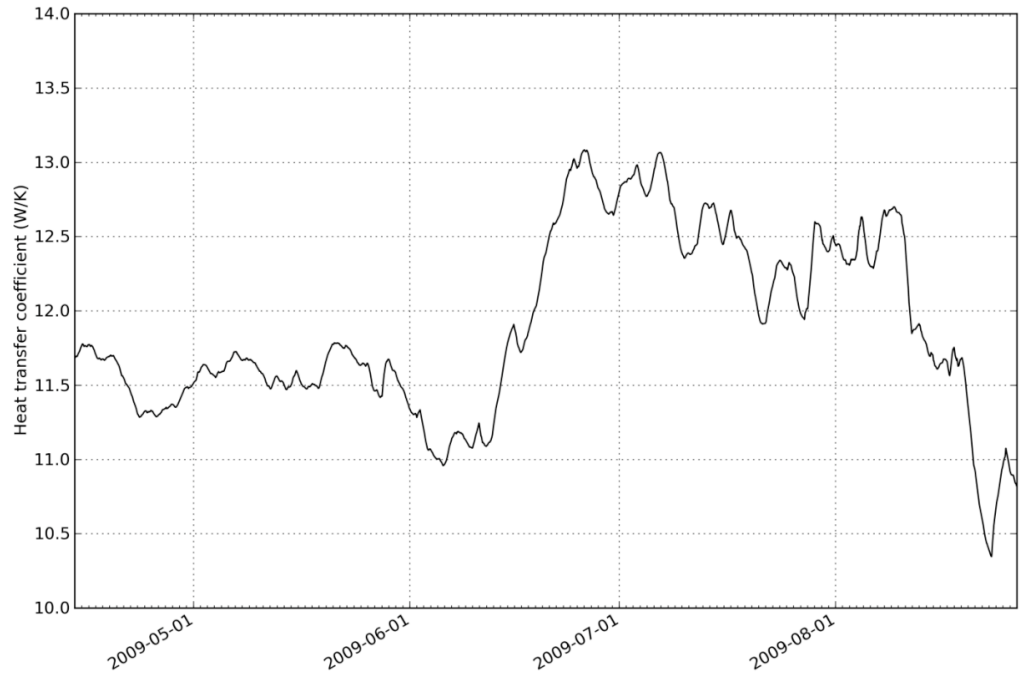

Figure 4. Thermal conductivity of the instrument-module during the winter period of darkness in 2009.

\section{FUEL EFFICENCY}

The total fuel efficiency of the PLATO diesel generator system is of great importance as it determines the fuel consumption and hence the length of time that PLATO can operate without solar assistance. Figure 5 shows the fuel level over the two and a half year period to date. Note that the fuel level has not been corrected for thermal expansion as there is currently no average fuel temperature measurement. The minor rise at the start of 2009 was when a small amount of new fuel was transferred and the engines replaced before the major refilling. Also note that in 2009 and 2010 , the fuel tank was filled slightly above the level sensor, resulting in the clipped peaks. 


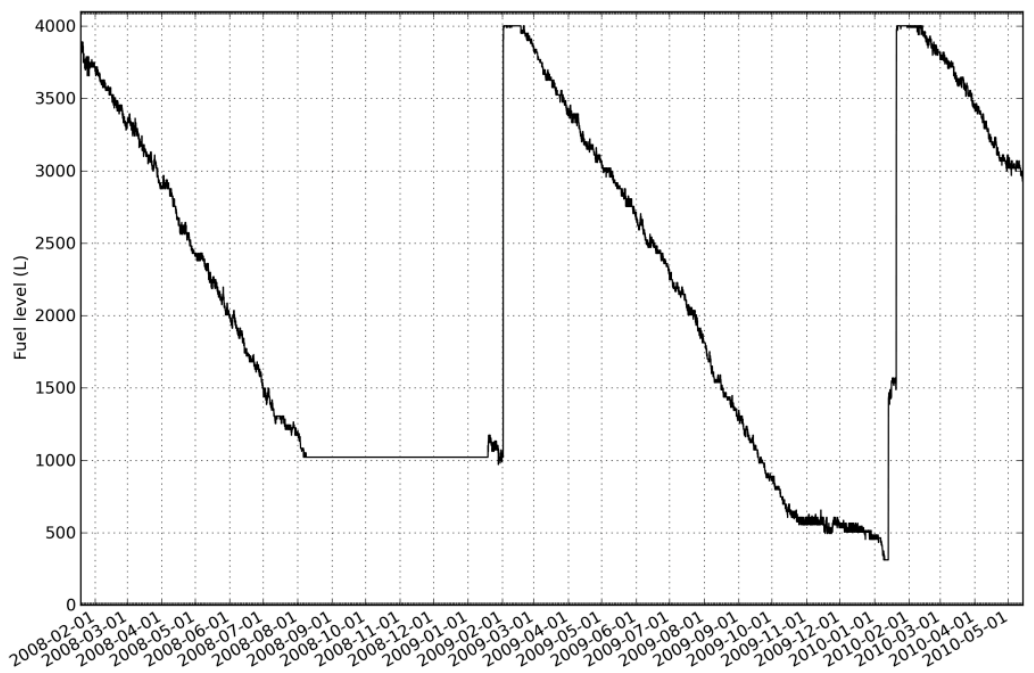

Figure 5. Engine module fuel level.

At the end of 2009, PLATO was put into a fuel-conserving strategy as all major dark observations had been completed. This can be seen in the reduced fuel consumption from mid October 2009 until PLATO was serviced in early 2010. Analyzing the period in 2009 where PLATO ran solely from engine power, the fuel consumption at a load of $600-$ $800 \mathrm{~W}$ was $0.79 \mathrm{~L} / \mathrm{kWh}$ of electrical output directly available for instruments. If the $80 \%$ efficiency of the DC/DC converter is taken into account (as its waste heat is not really wasted), this improves to $0.63 \mathrm{~L} / \mathrm{kWh}$ of useful power output.

Using the manufacturer's stated alternator efficiency of 0.9 and a density of the Jet-A1 fuel of $0.804 \mathrm{~g} / \mathrm{ml}$, the PLATO engines achieved an average fuel consumption of $460 \mathrm{~g} / \mathrm{kWh}$. The theoretical peak efficiency of the Hatz $1 \mathrm{~B} 30$ is $255 \mathrm{~g} / \mathrm{kWh}$ at our operating speed. The reduced efficiency of the $1 \mathrm{~B} 30$ compared to its optimum performance is most likely because we operated the engines at well below their optimum torque. Future improvements to the PLATO design will allow for the engines to run at their peak power, storing excess energy in a very high capacity battery bank. This will significantly improve the overall fuel efficiency. A detailed analysis of the engine performance at the altitude of Dome A can be found in Hengst et al $(2010)^{11,12}$.

\section{DATA TRANSFER PERFORMANCE}

Two Iridium satellite modems provide remote communication to PLATO. The two modems are fully independent and allow for full redundancy. Interactive control, software updates and instrument data transfer are performed by a suite of high efficiency custom utilities and protocols that can work within the constraints imposed by the 300 byte/s transfer rate of each modem.

Until servicing in 2010, one Iridium modem had a problem with its RF section, leading to low signal strength and frequent disconnections. During this period, the sustained maximum data transfer rate was $36.5 \mathrm{MB} / \mathrm{day}$. After the modem was replaced the instrument data rate increased to $45 \mathrm{MB} / \mathrm{day}$. The current maximum sustained data rate is $91 \%$ of the theoretical peak transfer rate. The data transfer system is flexible enough to allow for simple expansion by adding more modems, but currently is sufficient for control and retrieval of on-site reduced data to ensure the instrumentation is operating correctly. The latency of the connection is low enough to comfortably allow interactive sessions if required. The bulk data are stored on spinning and flash-memory hard drives and retrieved during the PLATO servicing missions.

\section{CONCLUSION}

The continued success of the PLATO observatory can be seen in the wealth of new scientific data and site-testing results that have started to emerge $e^{3,10,13}$. These types of observations require continued measurements over long time periods to provide accurate results for informed decisions on future instrumentation. Already a very significant telescope array, AST3 (Yuan et al 2010) ${ }^{14}$, is being built for Dome A based on the data acquired so far. The remoteness and extremely 
cold conditions are no longer an obstacle to the remote operation of instrumentation on the Antarctic plateau. This is demonstrated by PLATO's successful operation for over 730 days, of which the last 520 days have had 100\% uptime. It is the harsh conditions that make the Antarctic Plateau such an exceptional location for ground based observations, but PLATO shows that the difficulties of operating under these conditions can be overcome. Over the past two years, PLATO has been providing its instrumentation suite with the essential requirements of power, heat, control and communication. PLATO's fuel efficiency is lower than desired, and this is being addressed in the next generation of PLATO observatories currently under construction.

\section{ACKNOWLEDGEMENTS}

The authors wish to thank all members of the 2008, 2009 and 2010 Polar Research Institute of China expedition to Dome A for their heroic efforts in reaching the site and for the invaluable assistance they provided to the expedition astronomers in setting up the PLATO observatory and its instruments. This research is financially supported by the Australian Research Council, the Australian Antarctic Division, the Chinese Academy of Sciences, the National Natural Science Foundation of China, the US National Science Foundation, and the United States Antarctic Program.

\section{REFERENCES}

[1] Saunders, W., Lawrence, J. S., Storey, J. W. V., Ashley, M. C. B., Kato, S., Minnis, P., Winker, D. M., Liu, G., and Kulesa, C. "Where is the best site on Earth? Domes A, B, C and F, and Ridges A and B", Publ. Astron. Soc. Pac., 121, 976-992 (2009).

[2] Lawrence, J. S., Ashley, M. C. B., Tokovinin, A. and Travouillon, T., "Exceptional astronomical seeing conditions above Dome C in Antarctica," Nature, 431, 278-281 (2004).

[3] Yang, H., Kulesa, C. A., Walker, C. K., Tothill, N. F. H., Yang, J., Ashley, M. C. B., Cui, X., Feng, L., Lawrence, J. S., Luong-Van, D. M., Storey, J. W. V., Wang, L., Zhou, X., and Zhu, Z., "Exceptional terahertz transparency and stability above Dome A, Antarctica", Publ. Astron. Soc. Pac., 122, 490-494 (2010).

[4] Yuan, X., Cui ,X., Liu, G., Zhai, F., Gong, X, Zhang, R., Xia, L., Hu, J., Lawrence, J. S., Yan, J., Storey, J. W. V., Wang, L., Feng, L., Ashley, M. C. B., Zhou, X., Jiang Z., and Zhu, Z., "Chinese Small Telescope ARray (CSTAR) for Antarctic Dome A”, Proc. SPIE, 7012, 70124G - 70124G-8(2008).

[5] Bonner, C. S., Ashley, M. C. B., Lawrence, J. S., Luong-Van, D. M., and Storey, J. W. V., "Snodar: An acoustic radar for atmospheric turbulence profiling with 1m resolution", Acoustics Australia, 37, 4751 (2009).

[6] Moore, A., Allen, G., Aristidi, E., Ashley, M., Bedding, T., Beichman, C., Briguglio, R., Busso, M., Candidi, M., Ciardi, D., Cui, X., Cutispoto, G., Distefano, E., Espy, P., Everett, J., Feng, L., Hu, J., Jiang, Z., Kenyon, S., Kulesa, C., Lawrence, J., Le Roux, B., Leslie, T., Li, Y., Luong-Van, D., Phillips, A., Qin, W., Ragazzoni, R., Riddle, R., Sabbatini, L., Salinari, P., Saunders, W., Shang, Z., Stello, D., Storey, J., Sun, B., Suntzeff, N., Taylor, M., Tosti, G., Tothill, N., Travouillon, T., Van Belle, G., Von Braun, K., Wang, L., Yan, J., Yang, H., Yuan, X., Zhou, X., and Zhu, Z. "Gattini: a multisite campaign for the measurement of sky brightness in Antarctica”, Proc. SPIE, 7012, 701226 - 701226-10 (2008).

[7] Kulesa, C. A., Walker, C. K., Schein, M., Golish, D., Tothill, N., Siegel, P., Weinreb, S., Jones, G., Bardin, J., Jacobs, K., Martin, C. L., Storey, J., Ashley, M., Lawrence, J., Luong-Van, D., Everett, J., Wang, L., Feng, L., Zhu, Z., Yan, J., Yang, J., Zhang, X.-G., Cui, X., Yuan, X., Hu, J., Xu, Z., Jiang, Z., Yang, H., Li, Y., Sun, B., Qin, W., and Shang, Z., "Pre-HEAT: submillimeter site testing and astronomical spectra from Dome A, Antarctica”, Proc. SPIE, 7012, 701249-1 - 701249-11 (2008).

[8] Lawrence, J. S., Allen, G. R., Ashley, M. C. B., Bonner, C., Bradley, S., Cui, X., Everett, J. R., Feng, L., Gong, X., Hengst, S., Hu, J., Jiang, Z., Kulesa, C. A., Li, Y., Luong-Van, D., Moore, A. M., Pennypacker, C., Qin, W., Riddle, R., Shang, Z., Storey, J. W. V., Sun, B., Suntzeff, N., Tothill, N. F. H., Travouillon, T., Walker, C. K., Wang, L., Yan, J., Yang, J., Yang, H., York, D., Yuan, X., Zhang, X. G., Zhang, Z., Zhou, X., and Zhu, Z., "The PLATO Antarctic site testing observatory", Proc. SPIE, 7012, 701227-1 - 701227-12 (2008). 
[9] Luong-Van, D. M., Ashley, M. C. B., Everett, J. R., Lawrence, J. S., and Storey, J. W. V., "PLATO control and robotics", Proc. SPIE, 7019, 70192U-1 - 70192U-10 (2008).

[10] Yang, H., Allen, G., Ashley, M. C. B., Bonner, C. S., Bradley, S., Cui, X., Everett, J. R., Feng, L., Gong, X., Hengst, S., Hu, J., Jiang, Z., Kulesa, C. A., Lawrence, J. S., Li, Y., Luong-Van, D., McCaughrean, M. J., Moore, A. M., Pennypacker, C., Qin, W., Riddle, R., Shang, Z., Storey, J. W. V., Sun, B., Suntzeff, N., Tothill, N. F. H., Travouillon, T., Walker, C. K., Wang, L., Yan, J., Yang, J., York, D., Yuan, X., Zhang, X., Zhang, Z., Zhou, X. and Zhu, Z., "The PLATO Dome A Site-Testing Observatory: Instrumentation and First Results", Publ. Astron. Soc. Pac., 121, 174-184 (2009).

[11]Hengst, S., Allen, G. R., Ashley, M. C. B., Everett, J. R., Lawrence, J. S., Luong-Van, D. M., and Storey, J. W. V., "PLATO power: a robust low environmental impact power generation system for the Antarctic plateau", Proc. SPIE, 7012, 70124E-1 - 70124E-10 (2008).

[12]Hengst, S., Luong-Van, D. M., Everett, J. R., Lawrence, J. S., Ashley, M. C. B., Castel, D., and Storey, J. W. V., "A small, high-efficiency diesel generator for high-altitude use in Antarctica", International Journal of Energy Research, 34, 827-838 (2010).

[13]Zhou, X., Fan, Z., Jiang, Z., Ashley, M. C. B., Cui, X., Feng, L., Gong, X., Hu, J., Kulesa, C. A., Lawrence, J. S., Liu, G., Luong-Van, D. M., Ma, J., Moore, A. M., Qin, W., Shang, Z., Storey, J. W. V., Sun, B., Travouillon, T., Walker, C. K., Wang, J., Wang, L., Wu, J., Wu, Z., Xia, L., Yan, J., Yang, J., Yang, H., Yuan, X., York, D., Zhang, Z., and Zhu, Z., "The First Release of the CSTAR Point Source Catalog from Dome A, Antarctica", Publ. Astron. Soc. Pac., 122, 347-353 (2010).

[14] Yuan, X., Cui, X., Gong, X., Wang, D., and Yao, Z., "Progress of Antarctic Schmidt Telescopes (AST3) for Dome A", Proc. SPIE, 7733-63, in press (2010). 